\title{
Inherent Irreversibility of Hydromagnetic Third-Grade Reactive Poiseuille Flow of a Variable Viscosity in Porous Media with Convective Cooling
}

\author{
S. O. Salawu1 ${ }^{1 *}$, E. O. Fatunmbi ${ }^{2}$ \\ ${ }^{1 *}$ Department of Mathematics, University of Ilorin, Ilorin, Nigeria \\ e-mail: kunlesalawu2@gmail.com \\ ${ }^{2}$ Department of Mathematics and Statistics, Federal Polytechnic, Ilaro, Nigeria \\ e-mail: olusojiephesus@yahoo.com \\ *corresponding author
}

\begin{abstract}
The analysis of hydromagnetic inherent irreversibility of reactive third-grade poiseuille flow and incompressible fluid heat properties of a variable viscosity with convective cooling in fixed plates is investigated. The heat dissipation of reactive exothermic chemical in a uniform magnetic field moves past fluid in a porous medium in an irreversible mode and the entropy is created continually in the system within the channel stimulated by bimolecular chemical kinetic. The heat convective transfer at the walls surfaces with the immediate surrounding follows Newton's law of cooling. The dimensionless nonlinear equations are solved by the method of weighted residual (WRM). The results are used to obtain the Bejan number of the system and the entropy generation rate. The effects of the selected relevant parameters on the flow, entropy generation and Bejan number are demonstrated graphically and conferred with reverence to the parameters.
\end{abstract}

Keywords: Third-grade flow, entropy generation, poiseuille flow, Bejan number

\section{Introduction}

The flow of hydromagnetic fluid has drawn the attention of many scholars because of its significance in science and technology and application in mechanical designing manufacturing processes, atomic control engineering, dynamic of fluid astrophysical, aerodynamic of plasma, and magnetohydrodynamic (MHD) system (Cramer and Pai 1973, Dada and Salawu 2017). The leading work of (Hartmann 1837) is viewed as the root of MHD flow in a channel. The work examined the flow of an incompressible electrically conducting viscous flow fluid in parallel plates in the occurrence of a transverse magnetic field. The main features of this flow are that the magnetic field creates viscosity and thus makes the fluid inelastic and, for huge values of the Hartmann number, the fluid in the channel is divided into two sections, namely the central principal region and the boundary layer region (Hartmann and Lazarus 1937). The boundary layer area is restricted to thin boundary layers that arise close to the surfaces of the plates. This layer is identified as the Hartmann boundary layer. The variation in the rapidity and induced magnetic field is fast in the region. However, for the central core region, the induced magnetic field and velocity are nearly the same. 
It is important to note that the manner in which many flow fluids are utilized in engineering and industrial processes cannot be sufficiently clarified on the premise of the conventional Newtonian viscous model. Such includes polyphenylethers, dirt covering and suspensions, hydrocarbon oils, hot rolling, paper items, polyglycols and many others. A few constitutive formulations have been proposed to describe such a non-Newtonian flow fluid behavior. Among the several suggested models is that of third grade liquids for which one can sensibly plan to acquire a numerical solution. Broad investigations have been embraced which include such fluids (Yurusoy and Pakdemirli 2002, Makinde 2009). The compressive overall thermodynamics, uniqueness and stability of fluids of the differential kind with the fluids of third grade being an exceptional instance was considered by Rajagopal (1995). For problems including heat exchange for fluids of third grade, a general thermodynamics study of the constitutive function was carried out by Fosdick and Rajagopal (1980). A few investigations including heat and mass transport in viscoelastic third grade fluids were examined by Massoudi and Christe (1995), Makinde (2007a), Makinde (2007b). Previously, much consideration was given to flow in a channel for non-Newtonian liquids stimulated by the joined action of uniform velocity and axial pressure gradient on the wall in the occurrence of magnetic field (Hayat et al. 2004). Little consideration has been given to analyzing of non-Newtonian flow in a porous medium in the presence of buoyancy force problems.

Presently, entropy generation formulation has turned into a dynamic area of thermal engineering sciences since heat moves through liquids in an irreversible way and causes an undeniable change in entropy of the liquid particles. This is a result of a variation in the fluid particle temperature that prompts an irregular or scattered motion of the liquid particles. Obviously, this will diminish the effectiveness of the system. Inspired by the fact that several thermal procedures occur at a highertemperature, the important goal of this investigation was to analyze the thermal enactment of the channel flow problem examined before by Makinde and Chinyoka (2010). This is important to gauge the effectiveness of the configuration since raised entropy reduces the energy level of the system. The method here takes after the second law of thermodynamics that is centered on the first work of Bejan (1982) that has transformed the thermal optimization of engineering processes and utilized by Adesanya and Makinde (2015), Adesanya and Falade (2015), Adesanya and Makinde (2014) for viscoelastic fluids. The third grade liquid formulation with Vogel viscosity to examine the entropy production analysis in a pipe is investigated by Pakdemirli and Yilbas (2006). They demonstrated that distinctive viscosity parameters can either rise or diminish the entropy generation rate. A numerical solution for entropy impacts on convection flow in fixed walls was obtained by Chauhan and Kumar (2013). Recently, entropy generation and irreversibility of heat in a system have been studied for different flow configurations, as is archived in Siddiqui et al. (2010), Das and Jana (2014), Srinivas and Ramana (2016), Jangili et al. (2016), Prasad et al. (2014), Be'g et al. (2014).

The objective of the present study is to analyze the inherent irreversibility of third-grade reactive poiseuille flow of variable viscosity and incompressible electrically conducting fluid in a porous medium within fixed walls in the presence of a uniform transverse magnetic field with asymmetric convective cooling. The fluid flow mathematical model is formulated in Section 2. In Section 3, the weighted residual method (WRM) is presented. In Section 4, entropy generation is analyzed. In Section 5, the computational and graphical results are established and quantitatively discussed with respect to various embedded parameters in the flow system. 


\section{The flow mathematical formulation}

Consider laminar, hydromagnetic and isotropic third grade exothermic chemical reaction in a capricious viscosity of an incompressible fluid flow between two fixed parallel horizontal plates is demonstrated in Fig. 1. The non-Newtonian model is used to cause the viscoelastic effects in a porous medium. The flow is enhanced by bimolecular chemical kinetic taken to be driven by the mutual effects of the axial pressure gradient and buoyancy force. The flow is assumed to be in the direction of $x$ with $y$-axis normal to it. The fixed plates are subjected to convective exchange of heat with the ambient temperature.

Following from Makinde and Chinyoka (2010), neglecting reactive viscose fluid consumption and time-dependent influences. The momentum and energy balance equations governing model are as follows:

$$
\begin{aligned}
& -\frac{d \bar{P}}{d \bar{x}}+\frac{d}{d \bar{y}}\left[\bar{\mu}(T) \frac{d \bar{u}}{d \bar{y}}\right]+6 \lambda \frac{d^{2} \bar{u}}{d \bar{y}^{2}}\left(\frac{d \bar{u}}{d \bar{y}}\right)^{2}-\sigma_{0} B_{0}^{2} \bar{u}-\frac{\mu(T)}{K^{*}} \bar{u}+\rho g \beta\left(T-T_{0}\right)=0 \\
& k \frac{d^{2} T}{d \bar{y}^{2}}+\sigma_{0} B_{0}^{2} \bar{u}^{2}+\left(\frac{d \bar{u}}{d \bar{y}}\right)^{2}\left[\bar{\mu}(T)+2 \lambda\left(\frac{d \bar{u}}{d \bar{y}}\right)^{2}\right]+Q C A\left(\frac{K T}{v l}\right)^{m} e^{-\frac{E}{R T}}=0
\end{aligned}
$$

The boundary conditions imposed are:

$$
\begin{aligned}
& \bar{y}=b ; \quad \bar{u}=0,-k \frac{d T}{d \bar{y}}=h\left(T-T_{0}\right) \\
& \bar{y}=0 ; \quad \bar{u}=0, k \frac{d T}{d \bar{y}}=h\left(T-T_{0}\right)
\end{aligned}
$$

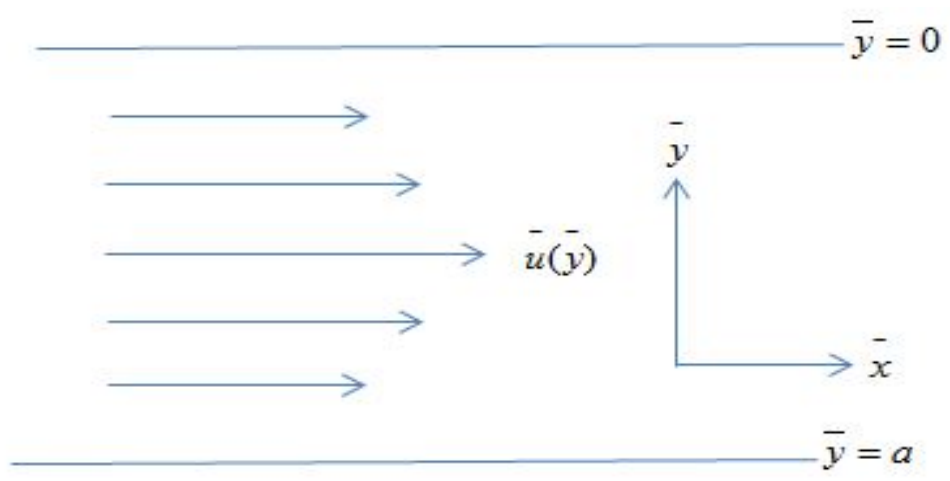

Fig. 1. Geometry of the flow

Here $\bar{u}, T_{0}, T, b, \bar{P}, K^{*}, \sigma_{0}, \rho, \lambda, B_{0}$ and $\beta$ are respectively the axial fluid velocity, temperature of the ambient, fluid temperature, channel width, fluid pressure, porosity term, electrical conductivity, density, material coefficients, electromagnetic induction and expansivity coefficient. The parameters $E, Q, C, l, k, h, K, m, R, A$ and $v$ are the activation energy, heat of reaction, initial species concentration, Planck's number, thermal 
conductivity, heat transfer coefficient, Boltzmann's constant, numerical exponent, universal gas constant, reaction rate constant and vibration frequency respectively.

The temperature dependent viscosity $(\bar{\mu})$ is defined to be $\bar{\mu}(T)=\mu_{0} e^{-c\left(T-T_{0}\right)}$

where $c$ is the viscosity variation term and $\mu_{0}$ is the initial fluid viscosity dynamic. Using the dimensionless quantities (4) in Eqs. (1)-(3) together with the temperature dependent viscosity, we obtain:

$$
\begin{aligned}
& y=\frac{\bar{y}}{b}, x=\frac{\bar{x}}{b}, u=\frac{\rho b \bar{u}}{\mu_{0}}, P=\frac{\bar{P} \rho b^{2}}{\mu_{0}^{2}}, \theta=\frac{E\left(T-T_{0}\right)}{R T_{0}^{2}}, \mu=\frac{\bar{\mu}}{\mu_{0}}, G=-\frac{d P}{d x}, \\
& G_{r}=\frac{\rho^{2} b^{3} g \beta R T_{0}^{2}}{\mu_{0}^{2} E}, r=\frac{c R T_{0}^{2}}{E}, \gamma=\frac{\lambda \mu_{0}}{\rho^{2} b^{4}}, \sigma=\frac{\mu_{0}^{3} e^{\frac{E}{R T}}}{\rho^{2} Q A b^{4} C}\left(\frac{v l}{R T_{0}}\right)^{m} \tau=\frac{R T_{0}}{E}, \\
& H^{2}=\frac{\sigma B_{0}^{2} b}{\mu_{0}}, B i=\frac{b h}{K}, \phi=\frac{b^{2}}{K}, \delta=\frac{Q E A b^{2} C e^{-\frac{E}{R T}}}{R K T_{0}^{2}}\left(\frac{K T_{0}}{v l}\right)^{m} .
\end{aligned}
$$

Therefore, the governing equations become:

$$
\begin{gathered}
G+e^{-r \theta} \frac{d^{2} u}{d y^{2}}-r e^{-r \theta} \frac{d \theta}{d y} \frac{d u}{d y}+6 \gamma \frac{d^{2} u}{d y^{2}}\left(\frac{d u}{d y}\right)^{2}-H^{2} u-\phi e^{-r \theta} u+G r \theta=0 \\
\frac{d^{2} \theta}{d y^{2}}+\delta(1+\tau \theta)^{m} e^{\frac{\theta}{1+\tau \theta}}+\sigma\left[H^{2} u^{2}+\left(\frac{d u}{d y}\right)^{2}\left(e^{-r \theta}+2 \gamma\left(\frac{d u}{d y}\right)^{2}\right)\right]=0
\end{gathered}
$$

The resultant boundary conditions are the following:

$$
\begin{aligned}
& u=0, \frac{d \theta}{d y}=-B i \theta \text { at } y=1 \\
& u=0, \frac{d \theta}{d y}=\operatorname{Bi\theta } \text { at } y=0
\end{aligned}
$$

where $G r, \delta, G, \gamma, \tau, \sigma, H, B i, \varphi$ and $\quad r$ represent the thermal Grashof number, Frankkamenetskii parameter, pressure gradient parameter, non-Newtonian parameter, activation energy parameter, viscous heating parameter, Hartmann number, Biot number, porosity parameter and variable viscosity parameter, respectively.

\section{Method of solution}

The idea of weighted residual method (Odejide and Aregbesola 2011, McGrattan 1998) is to look for an approximate result, in the polynomial form to the differential equation given as: 


$$
D[v(y)]=f \text { in the domain } R, A_{\mu}[v]=\gamma_{\mu} \text { on } \partial R
$$

where $D[v]$ represents a differential operator relating non-linear or linear spatial derivatives of the dependent variables $v, f$ is the function of a known position, $A_{\mu}[v]$ denotes the approximate number of boundary conditions with $R$ beeing the domain and $\partial R$ the boundary. By assuming an approximation to the solution $v(y)$, an expression of the form:

$$
v(y) \approx w\left(y, a_{1}, a_{2}, a_{3} \ldots a_{n}\right)
$$

which depends on a number of parameters $a_{1}, a_{2}, a_{3} \ldots a_{n}$ and is such that for an arbitrary value $a_{i}{ }^{\prime} s$ the boundary conditions are satisfied and the residual in the differential equation becomes:

$$
E\left(y, a_{i}\right)=L\left(w\left(y, a_{i}\right)\right)-f(y)
$$

The aim is to minimize the residual $E(y, a)$ to zero in some average sense over the domain. That is:

$$
\int_{Y} E(y, a) W_{i} d y=0 \quad i=1,2,3, \ldots n
$$

where the number of weight functions $W_{i}$ is exactly the same as the number of unknown constants $a_{i}$ in $w$. Here, the weighted functions are chosen to be Dirac delta functions. That is, $W_{i}(y)=\delta\left(y-y_{i}\right)$, such that the error is zero at the chosen nodes $y_{i}$. That is, the integration of Eq. (11) with $W_{i}(y)=\delta\left(y-y_{i}\right)$ results in $E\left(y, a_{i}\right)=0$.

By applying WRM to Eqs. (5) to (7), supposing a polynomial with unknown coefficients or parameters to be determined later, this polynomial is called the basis function which are taken as:

$$
u(y)=\sum_{i=0}^{n} a_{i} y^{i}, \quad \theta(y)=\sum_{i=0}^{n} b_{i} y^{i}
$$

By forcing the boundary conditions Eq. (7) on the basis functions Eq. (12) as well as using the basis functions on Eqs. (5) and (6) to get the residual, we have:

$$
\begin{aligned}
& u_{r}=G-H^{2}\left(y^{10} a_{10}+y^{9} a_{9}+y^{8} a_{8}+y^{7} a_{7}+y^{6} a_{6}+y^{5} a_{5}+y^{4} a_{4}+y^{3} a_{3}+y^{2} a_{2}+\right. \\
& \left.y a_{1}+a_{0}\right)+e^{-r\left(y^{10} b_{10}+y^{9} b_{9}+y^{8} b_{8}+y^{7} b_{7}+y^{6} b_{6}+y^{5} b_{5}+y^{4} b_{4}+y^{3} b_{3}+y^{2} b_{2}+y b_{1}+b_{0}\right)} \\
& \left(90 y^{8} a_{10}+72 y^{7} a_{9}+56 y^{6} a_{8}+42 y^{5} a_{7}+30 y^{4} a_{6}+20 y^{3} a_{5}+12 y^{2} a_{4}+6 y a_{3}+2 a_{2}\right)-\ldots
\end{aligned}
$$




$$
\begin{aligned}
& \theta_{r}=90 y^{8} b_{10}+72 y^{7} b_{9}+56 y^{6} b_{8}+42 y^{5} b_{7}+30 y^{4} b_{6}+20 y^{3} b_{5}+12 y^{2} b_{4}+6 y b_{3}+ \\
& 2 b_{2}+\delta\left(\tau \left(y^{10} b_{10}+y^{9} b_{9}+y^{8} b_{8}+y^{7} b_{7}+y^{6} b_{6}+y^{5} b_{5}+y^{4} b_{4}+y^{3} b_{3}+y^{2} b_{2}+\right.\right. \\
& \left.\left.y b_{1}+b_{0}\right)+1\right)^{m} e^{\frac{y^{10} b_{10}+y^{9} b_{9}+y^{8} b_{8}+y^{7} b_{7}+y^{6} b_{6}+y^{5} b_{5}+y^{4} b_{4}+y^{3} b_{3}+y^{2} b_{2}+y b_{1}+b_{0}}{r\left(y^{10} b_{10}+y^{9} b_{9}+y^{8} b_{8}+y^{7} b_{7}+y^{6} b_{6}+y^{5} b_{5}+y^{4} b_{4}+y^{3} b_{3}+y^{2} b_{2}+y b_{1}+b_{0}\right)+1}}+\ldots
\end{aligned}
$$

The residual errors are reduced to zero at some set of collocation points at a regular interval within the domain when $G r=0.2, m=0.5, B i=1.0, \tau=1.0, r=0.1, \sigma=1.0, \delta=0.5, \gamma=0.5$, $G=1.0, H=0.5$ and $\varphi=1.0$.That is, $y_{k}=\frac{(b-a) k}{N}$ where $k=1,2, \ldots, N-1$ and $a=0, b=1$, $N=10$. These are solved using MAPLE 2016 software to obtain the unknown coefficients.

Hence, the dimensionless momentum and energy equations become:

$$
\begin{aligned}
& u=-1.792943234 y^{10}+8.964716166 y^{9}-18.84836125 y^{8}+21.60514803 y^{7}- \\
& 14.82865225 y^{6}+6.519746541 y^{5}-1.911287621 y^{4}+0.2329047085 y^{3}- \\
& 0.3861075627 y^{2}+0.4448364769 y \\
& \theta=0.05502914456 y^{10}-0.2751457230 y^{9}+0.5743224003 y^{8}-0.6464152629 y^{7}+ \\
& 0.4347354227 y^{6}-0.1973648848 y^{5}+0.02496471423 y^{4}+0.07515235236 y^{3}- \\
& 0.5353187892 y^{2}+0.4900406258 y+0.4900406258
\end{aligned}
$$

The process of weighted residual method is repeated for different values of the parameters $G r, m, B i, \tau, r, \sigma, \delta, \gamma, G, H$ and $\varphi$.

\section{Entropy Generation Analysis}

The inclusive entropy generation equation per unit volume is define as:

$$
E_{G}=\frac{k}{T_{0}^{2}}(\nabla \bar{T})^{2}+\frac{\mu}{T_{0}} \Phi
$$

The first and second term of Eq. (17) are the heat transfer irreversibility and the viscous dissipation entropy generation respectively. Following from Eq. (17), the dimensionless entropy generation number is obtained as:

$$
N s=\frac{E^{2} b^{2} E_{G}}{R^{2} T_{0}^{2} k}=\left(\frac{d \theta}{d y}\right)^{2}+\frac{\delta \sigma}{\tau}\left\{H^{2} u^{2}+\left(\frac{d u}{d y}\right)^{2}\left[e^{-r \theta}+2 \gamma\left(\frac{d u}{d y}\right)^{2}\right]\right\}
$$

From Eq. (17), the first term is taken as $N_{1}$ while the second term is taken as $N_{2}$, i.e, 


$$
N_{1}=\left(\frac{d \theta}{d y}\right)^{2}, \quad N_{2}=\frac{\delta \sigma}{\tau}\left\{H^{2} u^{2}+\left(\frac{d u}{d y}\right)^{2}\left[e^{-r \theta}+2 \gamma\left(\frac{d u}{d y}\right)^{2}\right]\right\}
$$

The Bejan number $(\mathrm{Be})$ is mathematically defined as:

$$
\mathrm{Be}=\frac{N_{1}}{N s}=\frac{N_{1}}{N_{1}+N_{2}}=\frac{1}{1+\Phi}, \Phi=\frac{N_{2}}{N_{1}}
$$

The Ns and Be are illustrated respectively in the Figs. 12-19.

\section{Results and Discussion}

The related graphical results to Eqs. (5) - (7) are offered for different parameters values.

Figure 2 shows the effect of diverse values of Hartmann number $H$ on the velocity distributions. It is noticed from the figure that as the values of magnetic field parameter $H$ raise, the damping magnetic properties increase due to the presence of the Lorentz force, which results in increased resistance to flow and thereby retarded the convective fluid flow motion. Accordingly, the electrically conducting liquid takes a thrust from the magnetic force by influencing an electrically conducting fluid in the micro scale system.

Figure 3 represents the influence of porosity term $\varphi$ on the fluid flow profile. It is observed that the velocity flow fields reduce as the porosity parameter rises. The behavior is as a result of the wall of the plates that provides a supporting resistance to the flow fluid and causes the flow velocity to retard.

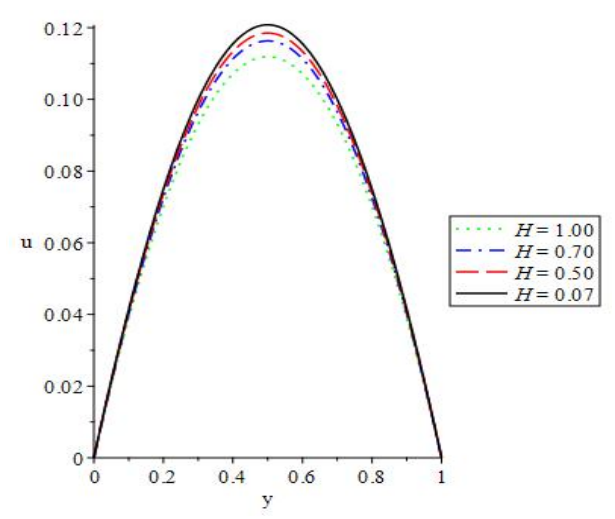

Fig. 2. Effects of $(H)$ on velocity

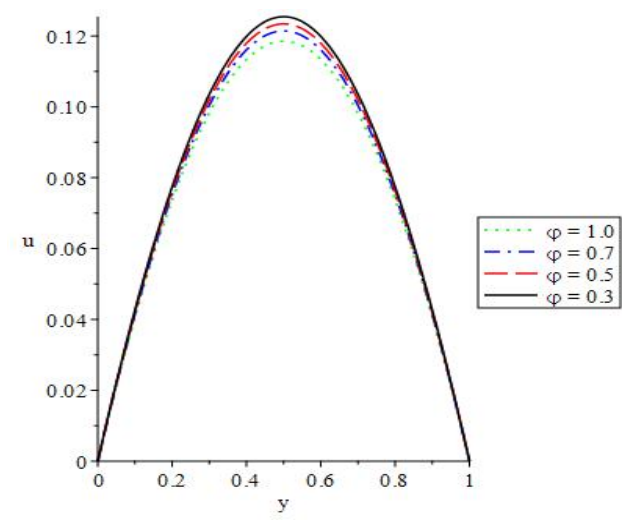

Fig. 3. Effects of $(\varphi)$ on velocity

Figures 4 and 5 depict the response of non-Newtonian parameter $\gamma$ on the fluid momentum and temperature profile. From the Figures, the fluid flow and the temperature distribution reduce as the viscoelastic material parameter increases. This is due to a rise in the fluid particle bonding force that makes the fluid to be more viscoelastic. Hence, the flow velocity and heat transfer in the system diminish. The falling trend is a result of the difference 
in the nonlinear heat at the plates and convective cooling, as the non-Newtonian parameter rises.

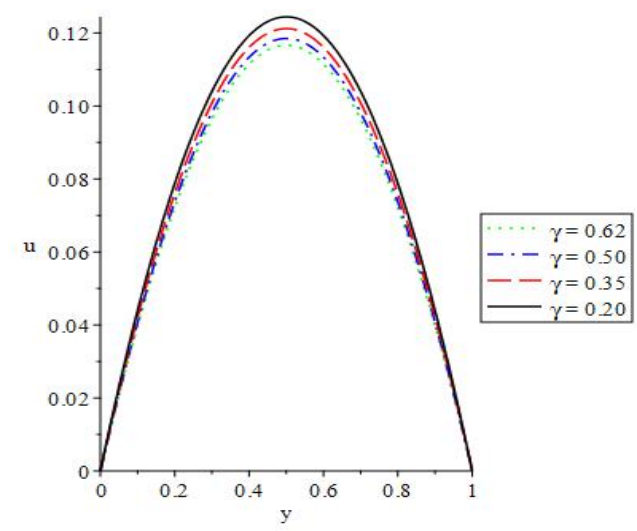

Fig. 4. Effects of $(\gamma)$ on velocity

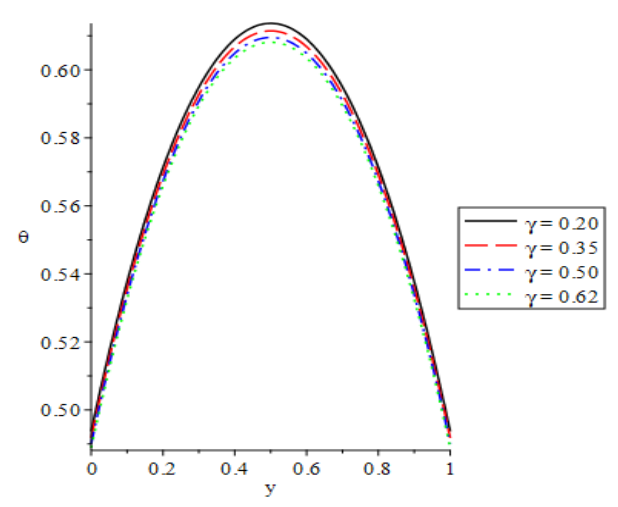

Fig. 5. Influences of $(\gamma)$ on temperature

Figure 6 illustrates the reaction of the flow velocity to difference in the variable viscosity parameter $r$. It can be seen that an increase in the parameter $r$ reduces the fluid viscosity and results in correspondingly diminishing the fluid's resistance to the flow. This fundamentally leads to strengthening in the fluid velocity, as demonstrated in the figure.

The strength of activation energy on the velocity profiles is presented in Fig. 7. Activation energy is the smallest energy which must exist in a chemical system with possible reactants to result in a chemical reaction. An increase in the parameter $\tau$ causes a reasonable rises in the heat transfer within the system. This is because of the presence of an appreciable number of molecules with translational energy equal to or greater than the activation energy.

The effect of the Frank-kamenetskii parameter $\delta$ on the temperature field is displayed in Fig. 8. An increase in the parameter values $\delta$ causes a noteworthy rise in the viscous heating source, which results in an increase in the reaction rate and accordingly boosts the temperature distributions. This is due to enhancement in the momentum viscosity coupling that results in rational rises in the flow temperature.

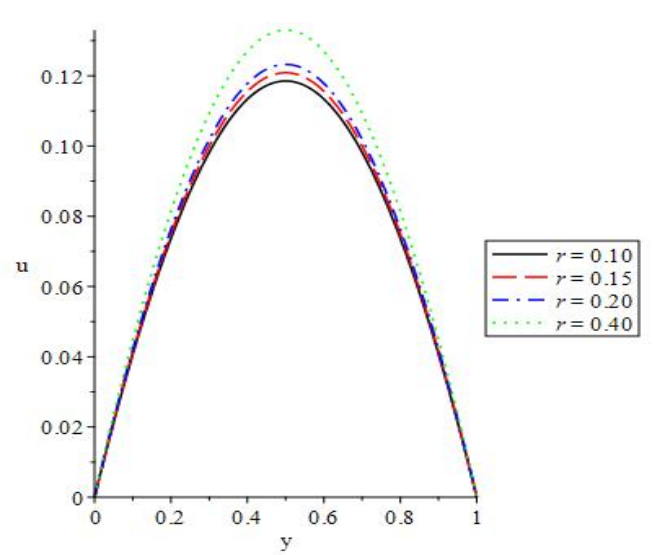

Fig. 6. Effects of $(r)$ on velocity

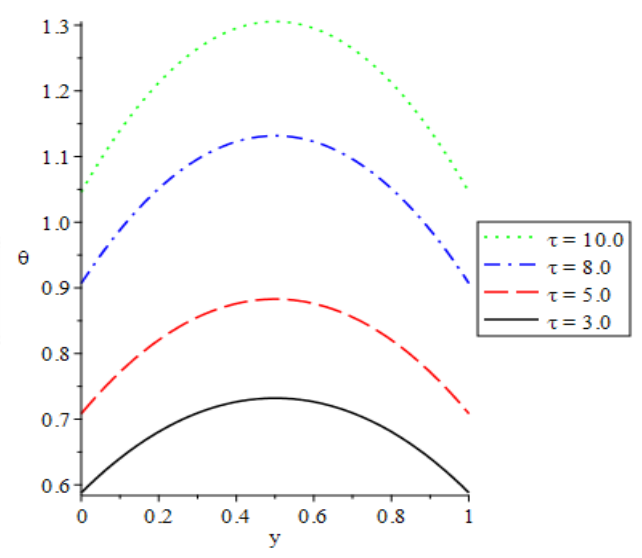

Fig. 7. Effects of $(\tau)$ on temperature 
Figure 9 shows the effect of Biot number $\mathrm{Bi}$ on the temperature fields. As it was noticed in the temperature boundary condition, the higher the Biot numbers the greater the convective cooling at the channel surfaces and thus congruently diminishes the temperatures at the surfaces and the bulk fluid. The entire temperature profile decreases with a rise in the Biot number as the fluid obstinately adjusts to the lower surface temperatures. The decline in the temperatures also reduces the fluid viscosity that in turn decreases the fluid momentum over the viscosity coupling that result in the pronounce changes in the heat distribution.

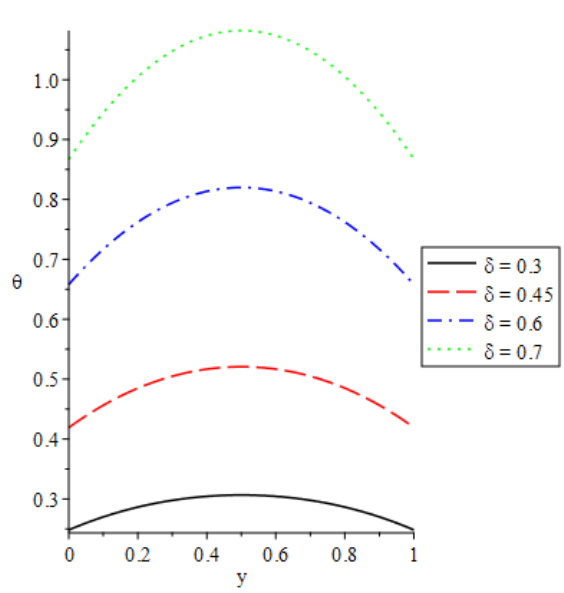

Fig. 8. Effects of $(\delta)$ on temperature

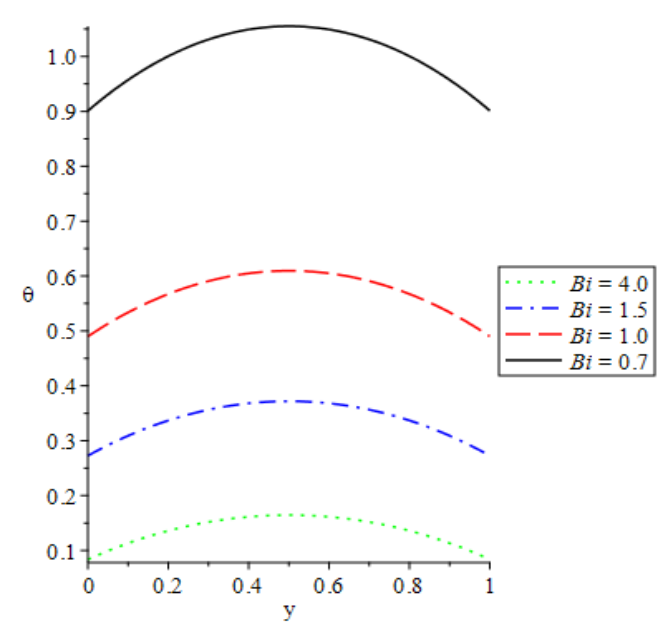

Fig. 9. Effects of $(B i)$ on temperature

The reaction of entropy generation rate to Biot number is exhibited in Fig. 10. It is noticed from the figure that a rise in the convective cooling $B i$ reduces the entropy production rate in the system. This is due to the irreversible heat flows from the warm surfaces to the ambient, in a manner that supports the Newtonian law of cooling. This causes the reduction in the entropy of the fluid region close to the cool surfaces. Lower thermal conductivity is associated to higher Biot number as well as to considerable cooling.

The effect of activation energy $\tau$ on entropy generation rate is portrayed in Fig. 11 . Activation energy is the smallest energy needed to start a chemical reaction process. A rise in the values of $\tau$ causes the chemical reaction to proceed at a reasonable rate and thereby increases the entropy generation in the system. This is because there is a reduction in the amount of heat that diffuses out of the system which leads to enhancement in the entropy produced in the system.

Figure 12 displays the effect of entropy generation rate to changing in the values of the Frank-kamenetskii parameter $\delta$. The figure shows that a rise in the reaction parameter enhances the entropy generation rate in the system. This is physically right as the internal heat generation increases, the reacting reagents enhance. The exothermic chemical relation raises the temperature transfer rate from the combustion area to the cool plates. Also, the heat is transported past the fluid to melt the fluid viscosity in other to increase the collision of particles; hence, extra heat is produced by the interaction of viscous fluid particle that in turn increases the entropy generation. 


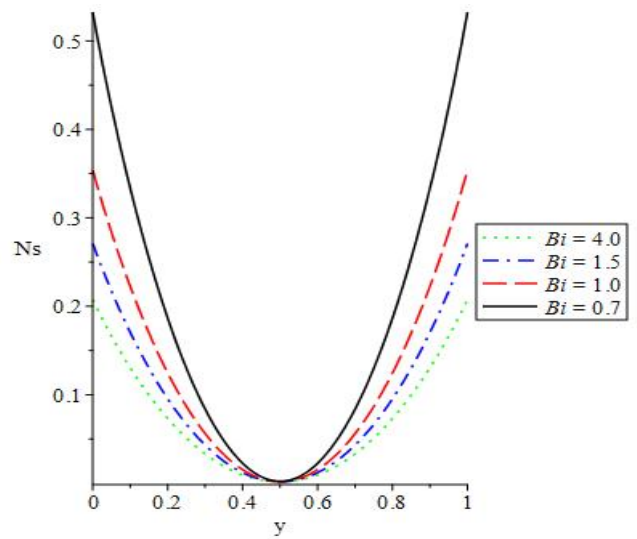

Fig. 10. Effects of (Bi) on entropy generation

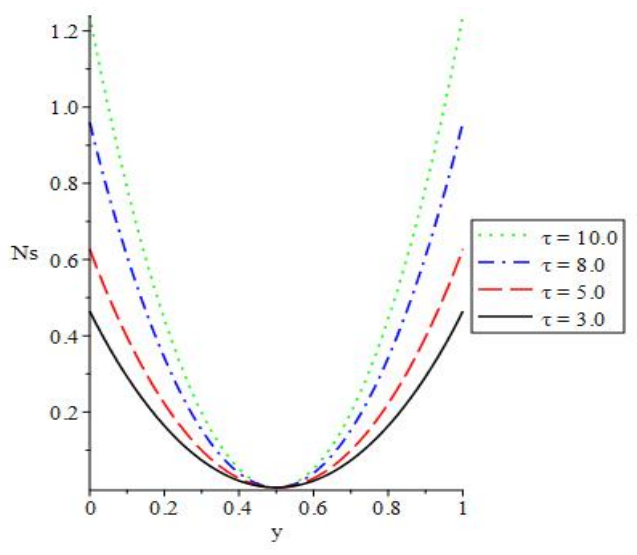

Fig. 11. Effects of $(\tau)$ on entropy generation

Figure 13 depicts the variation in Bejan number with changes in the values of Biot number. An increase in the convective cooling $B i$ is seen to strengthen the irreversibility of heat transfer in the system. The consequence specifies that heat transfer to the wall amplifies as the Biot number rises. Thus, irreversibility as a result of fluid friction reduces over heat transfer as the Biot number rises. Then, the Bejan number fields isdiminished.

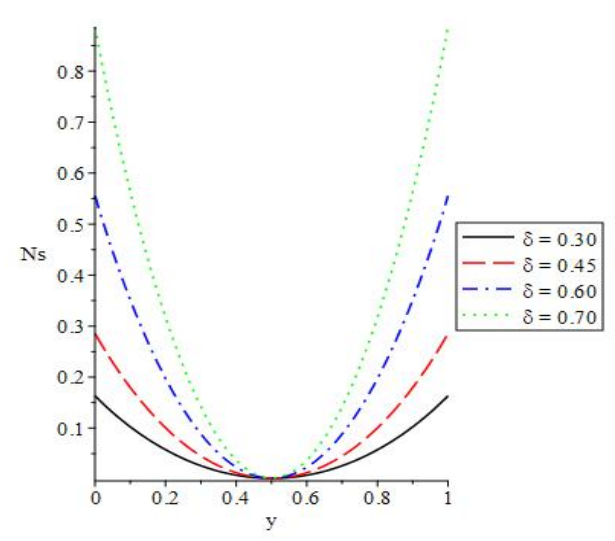

Fig. 12. Effects of $(\delta)$ on entropy generation

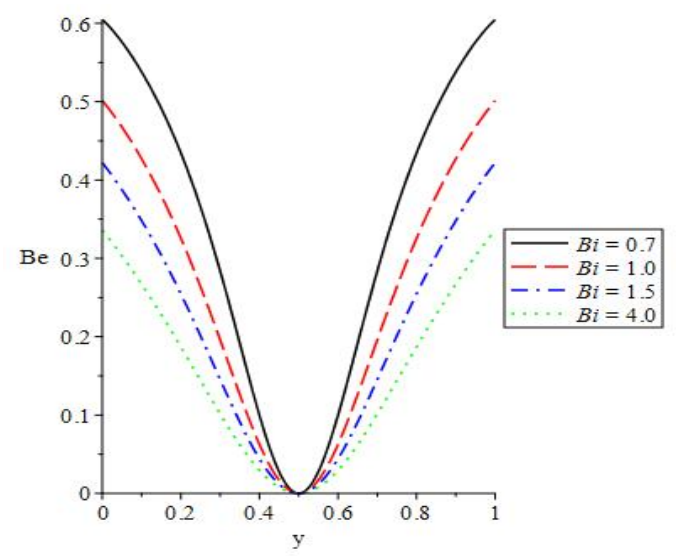

Fig. 13. Effects of $(B i)$ on Bejan number

The reaction of the Bejan number to an increase in the activation energy is illustrated in Fig. 14. It can be obtained from the figure that a variational increase in the activation energy leads to a corresponding increase in the heat transfer irreversibility rate of the system. This is due to the fact that the boundary layer get thinner as the parameter $\tau$ rises, thus the amount of irreversibility heat transport within the system to the fixed walls decreases and thereby results in an increase in the Bejan number profiles.

Figure 15 demonstrates the action of changing in the values of Frank-kamenetskii parameter $\delta$ on Bejan number. It is noticed that an increase in the parameter $\delta$ results in great rise in the irreversibility of heat transfer due to an exothermic chemical reaction that occurs in the fluid flow over a fixed channel. Thus, heat transfer rules over the irreversibility of the fluid 
friction as the bimolecular chemical kinetic effect is magnifies, thus increasing the Bejan number fields.

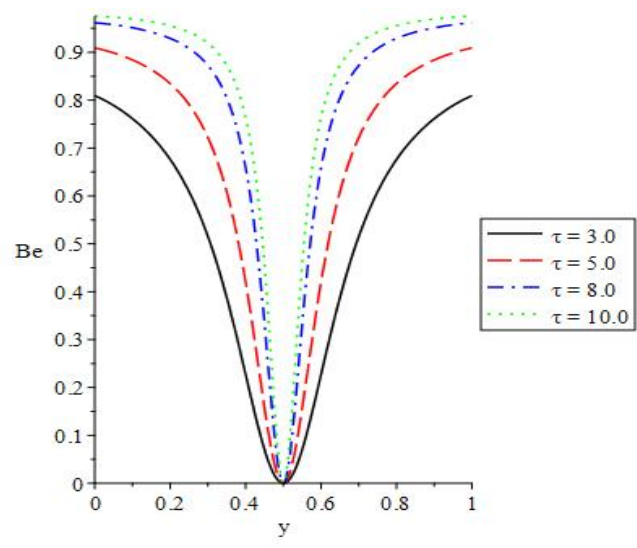

Fig. 14. Influences of $(\tau)$ on Bejan number

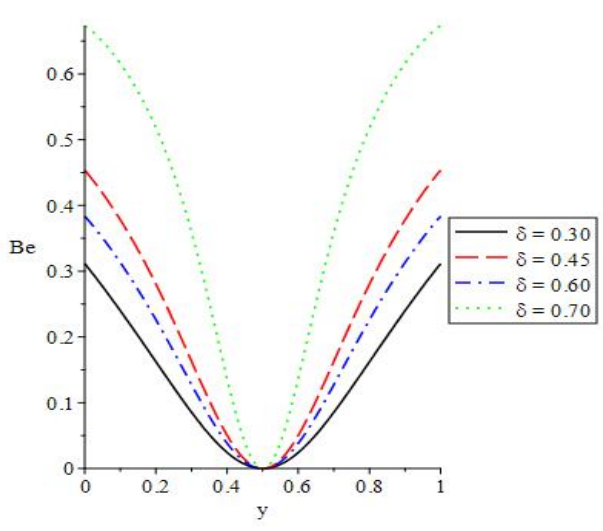

Fig. 15. Influences of $(\delta)$ on Bejan number

\section{Conclusion}

In the analysis, the irreversibility of heat in a chemical reaction of viscoelastic fluid past fixed walls with convective cooling was studied. This investigation was carried out using the second law of thermodynamics. The dimensionless momentum and energy equations were solved using weighted residual method. The solutions were used to obtain the velocity, temperature, entropy generation and Bejan number distribution. The study shows that:

(i) an increase in the non-Newtonian parameter increases the fluid flow velocity and the temperature profile. This is due to a rise in the fluid particle bonding force that makes the fluid to be more viscoelastic;

(ii) a rise in the Frank-Kamenetskii parameter needs to be observed as it contributes profoundly to damaging of the system thermo-fluid while the viscoelastic material effect and Biot number reduce the entropy profile;

(ii) a rise in the Frank-Kamenetskii was noticed to enhance the dominant irreversibility of heat transfer over fluid friction. Also, increasing the Biot number parameter declines fluid friction and increases heat transfer irreversibility in the channel.

\section{References}

Adesanya SO, Makinde OD (2015). Irreversibility analysis in a couple stress film flow along an inclined heated plate with adiabatic free surface, Physica A 432, 222-229.

Adesanya SO, Makinde OD (2014). Entropy generation in couple stress fluid flow through porous channel with fluid slippage, Int. J. Exergy 15, 344-362.

Adesanya SO, Falade JA (2015). Thermodynamics analysis of hydromagnetic third grade fluid flow through a channel filled with porous medium, Alexandria Eng. J. 54, 615-622.

Be'g OA, Uddin MJ, Rashidi MM, Kavyani N (2014). Double diffusive radiative magnetic mixed convective slip flow with Biot and Richardson number effects, J. Eng. Thermophy. 23, 79-97. 
Bejan A (1982). Entropy Generation through Heat and Fluid Flow, Wiley, New York.

Chauhan DS, Kumar V (2013). Entropy analysis for third-grade fluid flow with temperaturedependent viscosity in annulus partially filled with porous medium, Theor. Appl. Mech. 40 (3), 441-464.

Cramer KR, Pai SI (1973). Magnetofluid Dynamics for Engineers and Applied Physicists, McGraw Hill Book Company, New York.

Dada MS, Salawu SO (2017). Analysis of heat and mass transfer of an inclined magnetic field pressure-driven flow past a permeable plate, Appl. and Applied Math.: An Inter. Journal, 12 (1), 189-200.

Das S, Jana RN (2014). Entropy generation due to MHD flow in a porous channel with Navier slip, Ain Shams Eng. J. 5, 575-584.

Fosdick RL, Rajagopal KR (1980). Thermodynamics and stability of fluids of third grade, Proc. Roy. Soc. A 339, 351.

Hartmann J (1937). Theory of laminar flow of an electrically conducting liquid in a homogeneous magnetic field, Hg-Dynamics I, Math. Fys. Med. 15, 1-28.

Hartmann J, Lazarus F (1937). Experimental investigations on the flow of mercury in a homogeneous magnetic field, Hg-Dynamics II, Math. Fys. Med. 15, 145.

Hayat T, Nadeem S, Asghar S (2004). Hydromagnetic Couette flow of an Oldroyd-B fluid in a rotating system, Int. J. Engng. Sci. 42, 65-78.

Jangili S, Gajjela N, Anwar Beg O (2016). Mathematical modeling of entropy generation in magnetized micropolar flow between corotating cylinders with internal heat generation, Alexandria Eng. J. 55, 1969-1982.

Makinde OD (2007). Hermite-Padé approximation approach to thermal criticality for a reactive third-grade liquid in a channel with isothermal walls, Int. Commun. Heat Mass Transfer 34 (7), 870-877.

Makinde OD (2007). Thermal stability of a reactive third grade fluid in a cylindrical pipe: an exploitation of Hermite-Padé approximation technique, Appl. Math. Comput. 189, 690697.

Makinde OD (2009). Thermal criticality for a reactive gravity driven thin film flow of a third grade fluid with adiabatic free surface down an inclined plane, Appl. Math. Mech. 30 (3), 373-380.

Makinde OD, Chinyoka T (2010). Numerical study of unsteady hydromagnetic generalized couette flow of a reactive third-grade fluid with asymmetric convective cooling, Computers and Mathematics with Applications 61, 1167-1179.

Massoudi M, Christe I (1995). Effects of variable viscosity and viscous dissipation on the flow of a third grade fluid in a pipe, Int. J. Non-Linear Mech. 30, 687.

McGrattan ER (1998). Application of weighted residual methods to dynamic economics models, Federal Reserve Bank of Minneapolis Research Department Staff Report 232.

Odejide SA, Aregbesola YAS (2011). Applications of method of weighted residuals to problems with semi-finite domain, Rom. Journ. Phys., 56 (1-2), 14-24.

Pakdemirli M, Yilbas BS (2006). Entropy generation for pipe low of a third grade fluid with Vogel model viscosity, Int. J. Non-Linear Mech. 41 (3), 432-437.

Prasad VR, Abdul Gaffar S, Keshava Reddy E, Be'g OA (2014). Computational study of nonNewtonian thermal convection from a vertical porous plate in a non-Darcy porous medium with Biot number effects, J. Porous Media 17 (7), 601-622.

Rajagopal KR (1995). On Boundary Conditions for Fluids of the Differential Type: NavierStokes Equations and Related Non-Linear Problems, Plenum Press, New York, 273.

Siddiqui AM, Hameed M, Siddiqui BM, Ghori QK (2010). Use of Adomian decomposition method in the study of parallel plate flow of a third grade fluid, Commun. Nonlinear Sci. Numer. Simul. 15, 2388-2399. 
Srinivas J, Ramana Murthy JV (2016). Second law analysis of the flow of two immiscible micropolar fluids between two porous beds, J. Eng. Thermophys. 25 (1), 126-142.

Yurusoy M, Pakdemirli M (2002). Approximate analytical solutions for the flow of a third grade fluid in a pipe, Int. J. Non-Linear Mech. 37, 187 